\title{
Peak Inspiratory Flow Measured at Different Inhaler Resistances in Patients with Asthma
}

\author{
John Haughney, $\mathrm{MBChB}^{\mathrm{a}}$, Amanda J. Lee, $\mathrm{PhD}^{\mathrm{b}}$, Eddie McKnight, BSc ${ }^{\mathrm{c}}$, Inna Pertsovskaya, $\mathrm{PhD}^{\mathrm{c}}$, \\ Michelle O'Driscoll, BSc ${ }^{c}$, and Omar S. Usmani, MD, PhD ${ }^{\mathrm{d}}$ Glasgow, Aberdeen, Swaffham, and London, United Kingdom
}

What is already known about this topic? A patient's inspiratory flow together with the internal resistance of a dry powder inhaler provides the energy to disaggregate the drug formulation and allow effective drug delivery; however, misconceptions remain that inspiratory flow alone determines adequate dose delivery.

What does this article add to our knowledge? This study, using an In-Check DIAL, shows that most patients with asthma in the United Kingdom can generate sufficient inspiratory flow to use high-resistance dry powder inhalers. In addition, only approximately $70 \%$ achieve the recommended inspiratory flow rate to use a pressurized metered dose inhaler correctly.

How does this study impact current management guidelines? Clinicians can be reassured that most people with asthma can generate sufficient inspiratory flow to activate a range of dry powder inhalers, regardless of internal resistance. Further evidence of some patients' tendency to inhale "too fast" when using a pressurized metered dose inhaler is provided.

BACKGROUND: Patients' peak inspiratory flow rate (PIFR) may help clinicians select an inhaler device.

OBJECTIVE: To determine the proportion of patients with asthma who could generate correct PIFRs at different inhaler resistance settings.

METHODS: During a UK asthma review service, patients' PIFR was checked at resistance settings matching their current preventer inhaler device, at R5 (high-resistance dry powder inhaler [DPI]) and at R0 (low resistance, pressurized metered dose inhaler [pMDI]). Correct PIFR ("pass") was defined for R5

${ }^{a}$ Queen Elizabeth University Hospital, Glasgow, United Kingdom

${ }^{\mathrm{b}}$ Medical Statistics Team, University of Aberdeen, Aberdeen, United Kingdom

${ }^{c}$ National Services for Health Improvement Ltd, Swaffham, United Kingdom

${ }^{\mathrm{d}}$ National Heart and Lung Institute, Imperial College London and Royal Brompton Hospital, London, United Kingdom

The MAPLE Respiratory Service, which provided the specialist nurses for this study, is delivered by National Services for Health Improvement Ltd and funded by Orion Pharma (UK) Ltd as a service to medicine.

Conflicts of interest: J. Haughney has received honoraria or consultation fees from AstraZeneca, Boehringer, Cipla, Chiesi, Orion, Sanofi, and Teva. A. J. Lee received a consulting fee from National Services for Health Improvement to perform the statistical analyses. O. S. Usmani has received grants and personal fees from AstraZeneca, Boehringer Ingelheim, Chiesi, and GlaxoSmithKline and personal fees from Aerocrine, Cipla, Covis, Menarini, Mereo BioPharma, Mundipharma, Napp, Orion, Sandoz, and Takeda. The rest of the authors declare that they have no relevant conflicts of interest.

Received for publication July 22, 2020; revised September 3, 2020; accepted for publication September 14, 2020.

Available online October 1, 2020.

Corresponding author: John Haughney, MBChB, Queen Elizabeth University Hospital, Glasgow, UK. E-mail: j.haughney@abdn.ac.uk.

2213-2198

(C) 2020 The Authors. Published by Elsevier Inc. on behalf of the American Academy of Allergy, Asthma \& Immunology. This is an open access article under the CC BY-NC-ND license (http://creativecommons.org/licenses/by-nc-nd/4.0/).

https://doi.org/10.1016/j.jaip.2020.09.026 as 30 to $90 \mathrm{~L} / \mathrm{min}$ and for $\mathrm{R0}$ as 20 to $60 \mathrm{~L} / \mathrm{min}$. A logistic regression model examined the independent predictors of incorrect PIFR ("fail") at R5 and R0. Asthma severity was assessed retrospectively from treatment level.

RESULTS: A total of 994 adults (females 64.3\%) were included, of whom $90.4 \%$ currently used a preventer inhaler $\mathbf{7 1 . 5 \%}$ pMDI). PIFR pass rates were $93.7 \%$ at $\mathrm{R} 5$ compared with $70.5 \%$ at $\mathrm{RO}(P<.0001)$. All patients failing the R0 PIFR breathed in too fast $(>60 \mathrm{~L} / \mathrm{min})$, and $20 \%$ of patients currently using pMDI failed for this reason. Independent risk factors for failing R5 were female sex, older age group, and current preventer pMDI and for failing R0 included male sex, younger age group, current preventer DPI, and mild versus severe asthma.

CONCLUSIONS: This study demonstrates that most patients with asthma can achieve adequate inspiratory flow to activate high-resistance DPIs, whereas approximately a third of patients breathe in too fast to achieve recommended inspiratory flows for correct pMDI use, including one-fifth of patients who currently use a pMDI preventer. (c) 2020 The Authors. Published by Elsevier Inc. on behalf of the American Academy of Allergy, Asthma \& Immunology. This is an open access article under the CC BY-NC-ND license (http://creativecommons.org/licenses/bync-nd/4.0/). (J Allergy Clin Immunol Pract 2021;9:890-6)

Key words: Asthma; Inhaler resistance; Peak inspiratory flow rate

\section{INTRODUCTION}

Delivery of medications by inhalation is integral to the management of patients with chronic respiratory conditions and is preferred over systemic administration because of their rapid onset of action, high local drug concentrations achieved in the 
Abbreviations used

DPI-Dry powder inhaler

PIFR-Peak inspiratory flow rate

pMDI-Pressurized metered dose inhaler

lung, and fewer systemic side effects. ${ }^{1-3}$ The main classes of portable inhaled delivery systems are pressurized metered dose inhalers (pMDIs), dry powder inhalers (DPIs), and, most recently, soft mist inhalers. ${ }^{2,3}$ To be effective, all types of inhalers rely on correct inhaler technique and adherence to therapy; therefore, choosing the most appropriate device to suit individual patient needs together with clear guidance on their correct use are key elements to successful therapeutic outcomes. ${ }^{3,4}$

Breath-actuated DPIs deliver the drug to the respiratory tract as a result of the patient's inhalation triggering the device. ${ }^{5}$ The patient's inspiratory flow together with the internal resistance of the flow channel of the device provides the energy required to disaggregate the drug formulation and allow effective delivery to the lungs. ${ }^{5,6}$ Higher-resistance devices generate more turbulent flow, thus contributing proportionately more to the energy available for drug disaggregation, and therefore require less patient inspiratory flow to achieve adequate drug delivery. 5,6 Conversely, devices with lower internal resistance provide less manipulation of the patients' inspiratory flow and rely more on the patient generating higher inspiratory flows to activate the device. This suggests that patients with impaired lung function may benefit from using high-resistance devices. ${ }^{5} \mathrm{~A}$ minimum peak inspiratory flow rate (PIFR) of $30 \mathrm{~L} / \mathrm{min}$ is commonly required to activate DPI devices; that is, to trigger deaggregation and deliver the dose to the lungs. ${ }^{7-9}$ However, misconceptions remain that inspiratory flow alone dictates adequate dose delivery from DPIs and that some patients may not be able to generate sufficient PIFR to activate devices with high internal resistance. $^{6,10}$

An estimation of a patient's PIFR may help clinicians select a suitable inhaler device for patients with asthma. The In-Check DIAL is a simple tool to estimate PIFR across a range of resistances that reflect the resistances found in different inhaler devices. The aim of this prospective service evaluation study was to determine the proportion of patients with asthma who were able to generate a correct PIFR at resistance settings representative of "low-" to "high-"resistance devices using the In-Check device, and to describe the phenotypical features of these patients.

\section{METHODS}

\section{Study design and patient population}

This was a multicenter, prospective service evaluation study in which sequential patients were recruited during the conduct of the UK general practice asthma review service. The reviews were undertaken across 41 sites, conducted at each site by 1 of 10 specialist asthma nurses employed by National Services for Health Improvement Limited, between July 1, 2019, and October 31, 2019. Eligible patients were 5 years or older with a physician diagnosis of asthma confirmed by the presence of an asthma Read code. Read Codes are a coded thesaurus of clinical terms that are routinely used by clinicians in the UK National Health Service. ${ }^{11}$ Patients with chronic obstructive pulmonary disease, other significant respiratory disease, or cardiac conditions were excluded. This article presents the results for adults 18 years or older.

Because data were collected during routine service evaluation, with no randomized intervention, ethics committee approval and patient written informed consent were not required for this study. All data analyzed were completely anonymized.

\section{Patient assessments}

During their asthma review, each patient's PIFR was measured using the In-Check DIAL version G16 (Clement Clark International, Harlow, UK). All nurses had extensive experience in conducting asthma reviews and were trained collectively to standardize and optimize their use of the In-Check device. Inspiratory flow measurements were made according to a standard operating procedure at 2 or 3 resistance (R) levels:

1. The resistance corresponding to the patient's current principal preventer/controller inhaler device (if they used one, and if different from options 2 and 3 below)

2. The highest resistance setting (R5)

3. The lowest resistance setting, corresponding to pMDI (R0)

At each resistance setting tested, patients were instructed to breathe out to comfortable exhalation before performing up to 3 inhalation maneuvers. If the patient passed, this result was recorded. A "pass" was defined as a reading with the In-Check DIAL device of 30 to $90 \mathrm{~L} / \mathrm{min}$ at settings $\mathrm{R} 1$ to $\mathrm{R} 5$ or 20 to $60 \mathrm{~L} / \mathrm{min}$ at $\mathrm{R} 0$ setting, based on published standards ${ }^{9}$ (Table I). If the patient failed the assessment, 2 more attempts could be made. Patients could be taught simple technique and be given encouragement after every effort; for pMDI: "slow and gentle," and for DPI: "fast and hard." Hence, in total, each patient could do a maximum of 9 inhalation maneuvers.

Additional data items collected at asthma review or from patient medical records were age, sex, body mass index, smoking status, current principal preventer inhaler device, current principal reliever inhaler device, symptom score/control status, number of courses of oral corticosteroids in last 12 months, and peak expiratory flow. Asthma severity was assessed retrospectively from the level of treatment required to control symptoms and exacerbations, determined after the patient had been on controller treatment for at least 3 months, and defined as mild asthma (well controlled with as-needed inhaled short-acting beta-agonists or low-dose inhaled corticosteroids; Read code: 663V1); moderate asthma (well controlled with low-dose inhaled corticosteroid/long-acting beta 2 -agonist; Read code: 663V2); severe asthma (required moderate or high-dose inhaled corticosteroid/long-acting beta ${ }_{2}$-agonist \pm add-on therapy to maintain asthma control or remained uncontrolled despite this treatment; Read code: 663V3).

\section{Statistical analysis}

The sample size for this study was based on a convenience sample, with the aim of 1000 sequential patients completing the study assessments.

The proportion of patients able to achieve a correct PIFR (pass) at each resistance setting was described. Summary statistics (number [\%] for categorical factors and mean $\pm \mathrm{SD}$ for continuous factors) were produced for patient demographic and clinical characteristics and were used to describe the phenotypical features of those patients achieving a PIFR pass or fail.

The $\chi^{2}$ test was used to examine associations between pass rate at different resistance settings and each categorical demographic/clinical 
TABLE I. In-Check DIAL G16 inhaler resistance range ${ }^{9}$

\begin{tabular}{|c|c|c|}
\hline Inhaler device & $\begin{array}{l}\text { In-Check DIAL G16 } \\
\text { resistance setting* }\end{array}$ & $\begin{array}{l}\text { In-Check DIAL G16 flow } \\
\text { rate denoting clinically } \\
\text { effective range }\end{array}$ \\
\hline Handihaler & High & $30-90 \mathrm{~L} / \mathrm{min}$ \\
\hline $\begin{array}{l}\text { Easyhaler } \\
\quad \text { (monotherapy) }\end{array}$ & High & $30-90 \mathrm{~L} / \mathrm{min}$ \\
\hline NEXThaler & Medium high & 30-90 L/min \\
\hline Twisthaler & Medium high & $30-90 \mathrm{~L} / \mathrm{min}$ \\
\hline $\begin{array}{l}\text { Easyhaler } \\
\quad \text { (combination) }\end{array}$ & Medium high & $30-90 \mathrm{~L} / \mathrm{min}$ \\
\hline $\begin{array}{l}\text { Turbohaler } \\
\text { (Pulmicort) }\end{array}$ & Medium high & $30-90 \mathrm{~L} / \mathrm{min}$ \\
\hline $\begin{array}{l}\text { Turbohaler S } \\
\text { (Symbicort) }\end{array}$ & Medium & $30-90 \mathrm{~L} / \mathrm{min}$ \\
\hline Clickhaler & Medium & 30-90 L/min \\
\hline Spiromax & Medium & 30-90 L/min \\
\hline GenuAir & Medium & 30-90 L/min \\
\hline Ellipta & Medium low & 30-90 L/min \\
\hline Diskhaler & Medium low & 30-90 L/min \\
\hline Accuhaler & Medium low & $30-90 \mathrm{~L} / \mathrm{min}$ \\
\hline Breezhaler & Low & 30-90 L/min \\
\hline Respimat & pMDI/none & $20-60 \mathrm{~L} / \mathrm{min}$ \\
\hline pMDI & pMDI/none & $20-60 \mathrm{~L} / \mathrm{min}$ \\
\hline
\end{tabular}

*Comprises 5 representations of DPI device resistance (R5, High; R4, Medium high R3, Medium; R2, Medium low; R1, Low) and pMDI (R0).

factor. A linear trend test was applied where appropriate. In addition, a logistic regression model with forward conditional approach was used to identify statistically significant independent predictors of PIFR fail at each of R5 and R0. Hence, factors that did not statistically significantly predict failure do not appear in the final logistic model. For each independent predictor, a reference or base group was defined to enable interpretation of the risk of failure at R5 or R0 for each of the other levels (eg, using males as the reference group, the odds of failure at R5 for females can be interpreted as compared with that for males). The small number of patients who had missing values for 1 or more of the potential predictors were excluded from each logistic model in turn. Associations of predictors with failure rate were presented as odds ratio and associated 95\% CIs. Boxplots illustrating median (interquartile range) peak inspiratory flow at R5 and R0 using the last value of up to 3 inhalations and stratified across current preventer resistance groups were produced. All analyses were carried out using IBM SPSS Statistics for Windows version 25 (IBM Corp, Armonk, NY). Statistically significant results were defined as $P$ values less than or equal to .05 .

\section{RESULTS}

\section{Patient population}

A total of 1066 patients with asthma attending a routine asthma review service were evaluated, most of whom were adults ( $\mathrm{n}=994$ [93.2\%]) with 72 children (aged $\leq 17$ years). This report will focus on the 994 adults only.

Approximately two-third of the population comprised females, 56.6\% were aged between 40 and 69 years, with $26.8 \%$ older than 70 years, most patients were either
TABLE II. Demographic and clinical characteristics of the patients in the study

\begin{tabular}{|c|c|}
\hline Characteristic & $\begin{array}{c}\text { Patients with asthma } \\
\text { (N = 994) } \\
\end{array}$ \\
\hline \multicolumn{2}{|l|}{ Sex } \\
\hline Male & $355(35.7)$ \\
\hline Female & $639(64.3)$ \\
\hline \multicolumn{2}{|l|}{ Age group (y) } \\
\hline $18-24$ & $38(3.8)$ \\
\hline $25-39$ & $127(12.8)$ \\
\hline $40-54$ & $200(20.1)$ \\
\hline $55-69$ & $363(36.5)$ \\
\hline $70+$ & $266(26.8)$ \\
\hline \multicolumn{2}{|l|}{ Smoking status } \\
\hline Smoker & $106(10.7)$ \\
\hline Ex-smoker & $307(30.9)$ \\
\hline Never smoked & $581(58.5)$ \\
\hline \multicolumn{2}{|l|}{ BMI $\left(\mathrm{kg} / \mathrm{m}^{2}\right)$} \\
\hline$<18.5$ & $11(1.1)$ \\
\hline $18.5-24.9$ & $230(23.1)$ \\
\hline $25-29.9$ & $346(34.8)$ \\
\hline $30+$ & 405 (40.7) \\
\hline \multicolumn{2}{|l|}{ Missing, $\mathrm{n}=2$} \\
\hline \multicolumn{2}{|l|}{ Asthma severity } \\
\hline Mild & $461(46.4)$ \\
\hline Moderate & $468(47.1)$ \\
\hline Severe & $59(5.9)$ \\
\hline \multicolumn{2}{|l|}{ Missing, $\mathrm{n}=6$} \\
\hline \multicolumn{2}{|l|}{ Currently using preventer } \\
\hline Preventer inhaler device resistance: & $899(90.4)$ \\
\hline pMDI: R0 & $643(71.5)$ \\
\hline DPI: R1 (Low) & 0 \\
\hline R2 (Medium low) & $57(6.3)$ \\
\hline R3 (Medium) & $130(14.5)$ \\
\hline R4 (Medium high) & $44(4.9)$ \\
\hline R5 (High) & $25(2.8)$ \\
\hline
\end{tabular}

BMI, Body mass index.

Data presented as n (\%).

ex-smokers or never smokers (89.3\%), and $75.5 \%$ were classified as overweight or obese (Table II). Most patients were categorized as having either mild (46.4\%) or moderate (47.1\%) asthma, and $90.4 \%$ were currently being prescribed an asthma preventer therapy, most frequently administered via a pMDI (71.5\%).

\section{PIFR pass rates}

Of the whole adult study population of 994 patients, 931 patients $(93.7 \%)$ achieved a PIFR pass at resistance R5, the highest DPI resistance setting. Patients who failed to achieve a pass rate at this resistance were more likely to be female, of older age, and be a never smoker (Table III). Patients currently using a pMDI ( $\mathrm{n}=643$ ) were less likely to achieve a pass with R5 (91.6\%) compared with those 256 patients currently using a DPI (98.7\%). 
TABLE III. Pass rates at R5 resistance by demographic characteristics

\begin{tabular}{|c|c|c|}
\hline Characteristic & Pass rate, $\mathrm{n}(\%)$ & $P$ value* \\
\hline Total sample $(n=994)$ & $931(93.7)$ & \\
\hline \multicolumn{3}{|l|}{ Sex } \\
\hline Female & $589(92.2)$ & \\
\hline Male & $342(96.3)$ & .010 \\
\hline \multicolumn{3}{|l|}{ Age group (y) } \\
\hline $18-24$ & $38(100.0)$ & $<.001$ \\
\hline $25-39$ & $124(97.6)$ & \\
\hline $40-54$ & $193(96.5)$ & \\
\hline $55-69$ & $341(93.9)$ & \\
\hline $70+$ & $235(88.3)$ & \\
\hline BMI $\left(\mathrm{kg} / \mathrm{m}^{2}\right)$ & & .163 \\
\hline$<18.5$ & $10(90.9)$ & \\
\hline $18.5-24.9$ & $210(91.3)$ & \\
\hline $25-29.9$ & $327(94.5)$ & \\
\hline $30+$ & $382(94.3)$ & \\
\hline \multicolumn{3}{|l|}{ Missing, $\mathrm{n}=2$} \\
\hline Smoking status & & .037 \\
\hline Current smoker & $103(97.2)$ & \\
\hline Ex-smoker & $291(94.8)$ & \\
\hline Never smoked & $537(92.4)$ & \\
\hline Courses of OCS in past $12 \mathrm{mo}$ & & .787 \\
\hline 0 & $683(93.9)$ & \\
\hline 1 & $123(93.9)$ & \\
\hline $2+$ & $82(93.2)$ & \\
\hline \multicolumn{3}{|l|}{ Missing, $\mathrm{n}=48$} \\
\hline Severity of asthma & & .168 \\
\hline Mild & $426(92.4)$ & \\
\hline Moderate & $443(94.7)$ & \\
\hline Severe & $56(94.9)$ & \\
\hline \multicolumn{3}{|l|}{ Missing, $\mathrm{n}=6$} \\
\hline Current preventer resistance & & .002 \\
\hline No current preventer & $89(93.7)$ & \\
\hline $\mathrm{R} 0 / \mathrm{pMDI}$ & $589(91.6)$ & \\
\hline R2 (Medium low) & $57(100)$ & \\
\hline R3 (Medium) & $129(99.2)$ & \\
\hline R4 (Medium high) & $42(95.5)$ & \\
\hline R5 (High) & $25(100)$ & \\
\hline
\end{tabular}

$B M I$, Body mass index; OCS, oral corticosteroid.

*Across categories from $\chi^{2}$ linear trend test.

In the whole adult study population, the number of patients achieving a pass rate at the R0 (pMDI) setting was 701 (70.5\%), statistically significantly fewer than those achieving a pass rate at R5 $(P<.0001)($ Table IV). The reason for all patients failing the R0 test was their PIFR being more than $60 \mathrm{~L} / \mathrm{min}$. Patients failing to achieve a pass rate at R0 were more likely to be male and to be using a higher-resistance preventer. Patients currently using a pMDI $(n=643)$ were more likely to achieve a pass at R0 $(80.2 \%)$ compared with those currently using a DPI $(\mathrm{n}=256$ [38.9\%]). With respect to their current inhaler, only 4 of 256 patients (1.2\%) currently using a DPI failed at the same resistance as their current device. Pass rates by current device
TABLE IV. Pass rates at RO resistance by demographic characteristics

\begin{tabular}{|c|c|c|}
\hline Characteristic & Pass rate, $\mathrm{n}(\%)$ & $P$ value* \\
\hline Total sample $(\mathrm{n}=991)$ & $701(70.5)$ & - \\
\hline Sex & & .013 \\
\hline Female & $467(73.4)$ & \\
\hline Male & $234(65.9)$ & \\
\hline \multicolumn{3}{|l|}{ Missing, $\mathrm{n}=3$} \\
\hline Age group (y) & & .135 \\
\hline $18-24$ & $28(73.7)$ & \\
\hline $25-39$ & $80(63.0)$ & \\
\hline $40-54$ & $136(68.0)$ & \\
\hline $55-69$ & $269(74.1)$ & \\
\hline $70+$ & $188(71.5)$ & \\
\hline \multicolumn{3}{|l|}{ Missing, $\mathrm{n}=3$} \\
\hline BMI $\left(\mathrm{kg} / \mathrm{m}^{2}\right)$ & & .676 \\
\hline$<18.5$ & $8(72.7)$ & \\
\hline $18.5-24.9$ & $160(69.9)$ & \\
\hline $25-29.9$ & $242(70.1)$ & \\
\hline $30+$ & $289(71.5)$ & \\
\hline \multicolumn{3}{|l|}{ Missing, $\mathrm{n}=5$} \\
\hline Smoking status (adults) & & .218 \\
\hline Current smoker & $67(63.8)$ & \\
\hline Ex-smoker & 219 (71.6) & \\
\hline Never smoked & $415(71.6)$ & \\
\hline Courses of OCS in past $12 \mathrm{mo}$ & & .424 \\
\hline 0 & $528(72.8)$ & \\
\hline 1 & $80(61.1)$ & \\
\hline $2+$ & $63(72.4)$ & \\
\hline \multicolumn{3}{|l|}{ Missing, $\mathrm{n}=51$} \\
\hline Severity of asthma & & .804 \\
\hline Mild & $337(73.1)$ & \\
\hline Moderate & $311(66.9)$ & \\
\hline Severe & $50(84.7)$ & \\
\hline \multicolumn{3}{|l|}{ Missing, $\mathrm{n}=9$} \\
\hline Current preventer resistance & & $<.001$ \\
\hline No current preventer & $72(75.8)$ & \\
\hline R0/pMDI & $516(80.2)$ & \\
\hline R2 (Medium low) & $25(44.6)$ & \\
\hline R3 (Medium) & $67(51.5)$ & \\
\hline R4 (Medium high) & $15(35.7)$ & \\
\hline R5 (High) & $6(24.0)$ & \\
\hline Missing, $\mathrm{n}=3$ & & \\
\hline
\end{tabular}

$B M I$, Body mass index; OCS, oral corticosteroid.

*Across categories from $\chi^{2}$ linear trend test.

resistance were $100 \%(n=25$ of 25$)$ for $\mathrm{R} 5,100 \%(\mathrm{n}=44$ of 44) for R4, 98.5\% (128 of 130) for R3, and 96.5\% ( $n=55$ of 57) for R2. No patients had a current device with R1 resistance. In comparison, $19.8 \%$ of patients who currently used a pMDI $(\mathrm{n}=643)$ failed to achieve a pass rate at $\mathrm{R} 0$ resistance.

\section{Independent predictors of PIFR failure rates}

A forward conditional logistic regression model showed that the independent risk factors of PIFR measurement failure at resistance R5 were sex, age group, and resistance of current preventer (Table V). Females were 2.5 times more at risk of 
TABLE V. Independent demographic and clinical predictors of failure rate at $\mathrm{R} 5$ and $\mathrm{R} 0$ resistance

\begin{tabular}{|c|c|c|}
\hline $\begin{array}{l}\text { Demographic and clinical } \\
\text { characteristic }\end{array}$ & $\begin{array}{c}\text { Failure rate at } \mathrm{RO} \text {, } \\
\text { odds ratio } \\
(95 \% \mathrm{Cl})\end{array}$ & $\begin{array}{c}\text { Failure rate } \\
\text { at } \mathrm{R} 5,^{*} \text { odds ratio } \\
(95 \% \mathrm{Cl})\end{array}$ \\
\hline \multicolumn{3}{|l|}{ Sex } \\
\hline Male & Reference & Reference \\
\hline Female & $0.59(0.43-0.81)$ & $2.46(1.27-4.76)$ \\
\hline \multicolumn{3}{|l|}{ Age group (y) } \\
\hline$\leq 39$ & Reference & Reference \\
\hline $40-54$ & $0.91(0.55-1.49)$ & $1.79(0.45-7.09)$ \\
\hline $55-69$ & $0.55(0.35-0.87)$ & $3.25(0.94-11.22)$ \\
\hline$\geq 70$ & $0.71(0.44-1.14)$ & $6.12(1.81-20.77)$ \\
\hline \multicolumn{3}{|l|}{$\begin{array}{c}\text { Current preventer } \\
\text { resistance }\end{array}$} \\
\hline No current preventer & $1.10(0.64-1.89)$ & $0.82(0.31-2.14)$ \\
\hline R0/pMDI & Reference & Reference \\
\hline R2 (Medium low) & $5.46(2.85-10.45)$ & - \\
\hline R3 (Medium) & $5.27(3.29-8.43)$ & $0.08(0.01-0.59)$ \\
\hline R4 (Medium high) & $8.85(4.37-17.93)$ & $0.50(0.12-2.16)$ \\
\hline R5 (High) & $12.93(4.78-34.92)$ & - \\
\hline Severity of asthma & & - \\
\hline Mild & Reference & \\
\hline Moderate & $0.76(0.52-1.12)$ & \\
\hline Severe & $0.21(0.09-0.51)$ & \\
\hline $\begin{array}{l}\text { No. of courses of OCS in } \\
\text { last } 12 \mathrm{mo}\end{array}$ & & - \\
\hline 0 & Reference & \\
\hline 1 & $1.90(1.24-2.93)$ & \\
\hline $2+$ & $1.29(0.73-2.28)$ & \\
\hline
\end{tabular}

$B M I$, Body mass index; OCS, oral corticosteroid.

Factors considered for entry into each logistic model were sex, age group, BMI group, smoking status, severity of asthma severity, number of courses of OCS in past $12 \mathrm{mo}$, and current preventer resistance. A forward stepwise procedure was used to identify independent predictors of failure; factors that did not independently predict failure are excluded from the model estimates shown.

No patients with an R2 medium low or an R5 high preventer failed at R5 resistance. *A total of 59 patients were excluded from the failure rate at the R5 model and 56 from the R0 model because of missing values for 1 or more of the 7 potential predictors.

failing the measurement at R5 compared with male patients, and patients 40 years or older were more at risk of failing versus those 39 years or younger, with statistically significantly reduced odds shown in patients 70 years or older whose risk of failing was 6 times greater than of patients 39 years or younger (odds ratio, 6.12; 95\% CI, 1.81-20.77). Compared with patients who currently used a pMDI, patients currently using a medium-resistance DPI (R3) had significantly reduced odds for failing the R5 measurement (odds ratio, 0.08 ; $95 \% \mathrm{CI}$, 0.01-0.59).

The logistic regression model evaluating independent predictors of failure at R0 showed that females had significantly reduced odds of failure compared with males (odds ratio, 0.59; $95 \% \mathrm{CI}, 0.43-0.81$ ), and patients 40 years or older were less at risk of failing versus those 39 years or younger, the difference being statistically significant in patients aged 55 to 69 years (odds ratio, 0.55; 95\% CI, 0.35-0.87) (Table V). Patients with severe asthma were significantly less likely to fail than those with mild asthma (odds ratio, 0.21; 95\% CI, 0.09-0.51). Compared with patients who currently used a pMDI, patients using a R2 preventer had more than 5 times the odds (odds ratio, 5.46; 95\% CI, 2.85-10.45) of failing the R0 measurement, which increased to 12 times the risk of failing among those currently using an R5 inhaler (odds ratio, 12.93; 95\% CI, 4.78-34.92).

Figure 1 shows the distribution of peak inspiratory flow at R5 and R0 resistance across different current preventer inhalers. Within each preventer group, there was a wide range of values. Against R0 resistance, patients currently using DPIs fared worse than those currently using a pMDI, with a trend toward higher PIFR with increasing resistance in their current inhaler. Against R5 resistance there was a more homogeneous outcome regardless of current inhaler.

\section{DISCUSSION}

This study showed that, irrespective of their current device and with the same level of training as given at a regular asthma review service, most patients with asthma had the capacity to achieve the PIFR required to use a high-resistance DPI (R5), and nearly all patients met the PIFR requirements for their current DPI device. In comparison, approximately $30 \%$ of the total adult patient population evaluated failed to achieve a PIFR pass rate with the pMDI device (R0). In all patients failing the R0 test, a PIFR greater than $60 \mathrm{~L} / \mathrm{min}$ was generated; that is, they were inhaling too fast. Of these failures, roughly $60 \%$ were current DPI users and $40 \%$ were current pMDI users. However, nearly $20 \%$ of patients who currently used a pMDI device failed with R0 even following 3 attempts with coaching.

The factors required to achieve correct inhaler use are well recognized and rely on appropriate training and reinforcement. ${ }^{12,13}$ These include correct preparation/handling of the device, a gentle full exhalation away from the mouthpiece before inhaling, ensuring a good mouth seal around the mouthpiece, a correct inhalation maneuver ("deep and forceful from the start" for DPI and "slow and steady over 4-5 seconds" for pMDI) followed by a breath hold for at least 5 seconds. ${ }^{12-14}$ Our study examined only PIFR, with the support and guidance of nurses to overcome technique errors. A marked observation in this study was the number of patients failing to achieve the required PIFR for correct pMDI use due to inhaling too fast. Rapid inhalation with a pMDI is associated with increased oropharyngeal deposition and suboptimal drug delivery, ${ }^{12,13}$ which has potential clinical implications. A previous study evaluating inhaler technique and asthma control as part of the UK IMPACT (Improving the Management of Patients Asthma and COPD Treatment) asthma review service also reported that approximately a third of patients failed the pMDI inspiratory flow criteria $(10-50 \mathrm{~L} / \mathrm{min})$ after training and showed a statistically significant association between pMDI misuse (inhaler technique measurements included inspiratory flow, synchronization of flow/actuation, and breath hold) and poor asthma control. ${ }^{15}$ Giraud and Roche, ${ }^{16}$ in a general practitioner study, similarly reported that $34 \%$ of adult patients failed the "slow inspiratory flow" criterion and found an association between pMDI misuse (combined criteria) and asthma instability. The CRITIKAL (CRITical Inhaler mistaKes and Asthma controL) study, based on an asthma review service in Australia and 7 European countries including the United Kingdom, reported that errors related to inspiratory effort were one of the most common errors made by patients using the Turbohaler (32\%), Diskus (38\%), and 


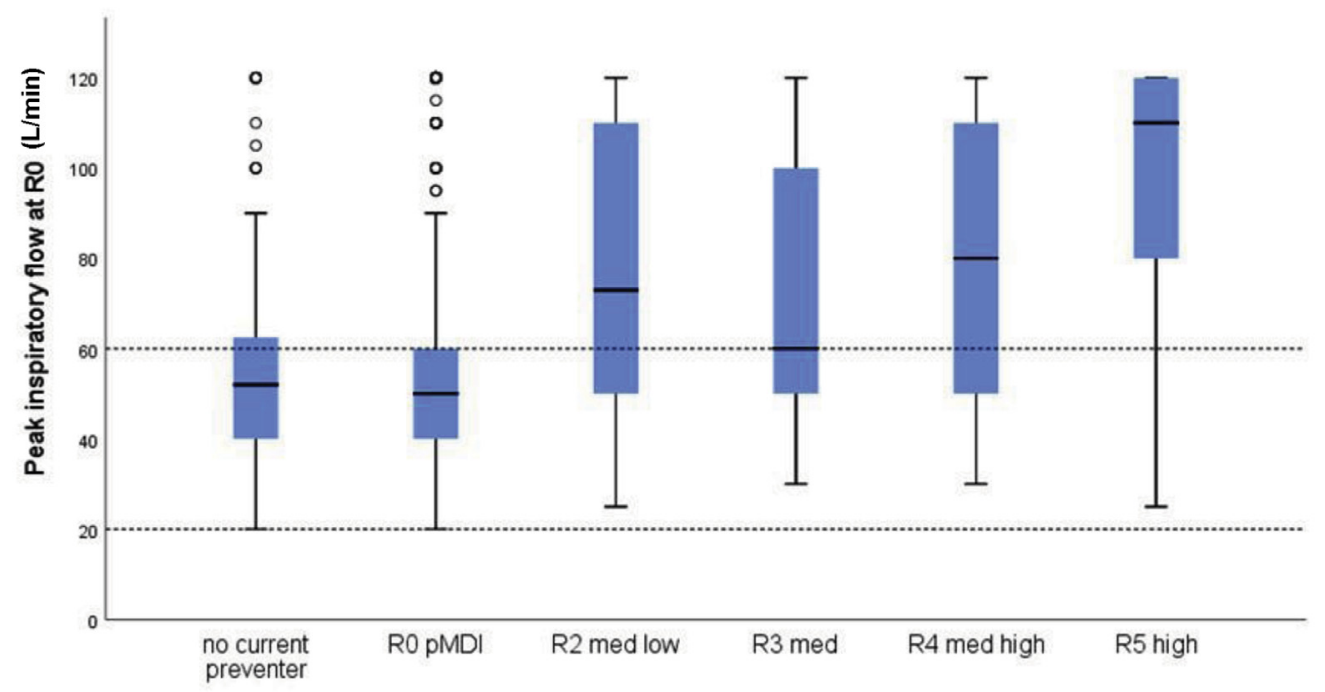

A

Current preventer resistance

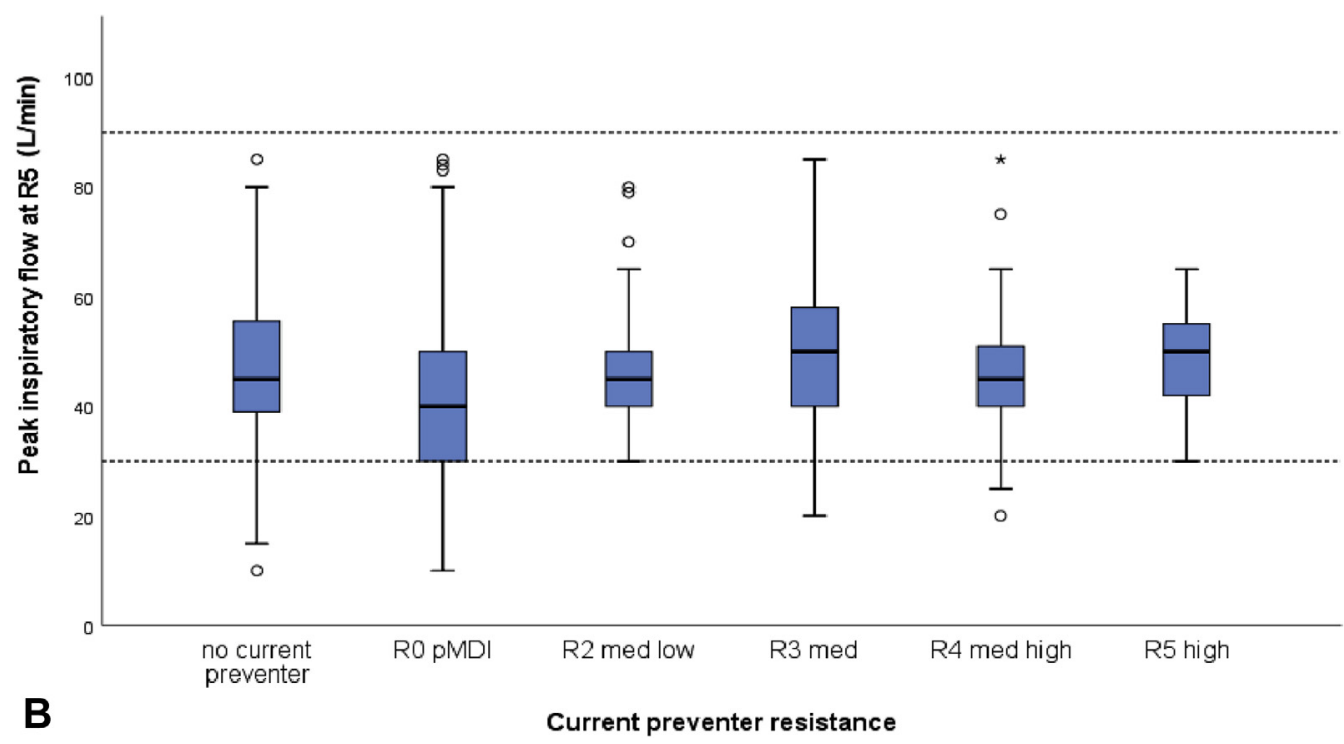

FIGURE 1. Boxplot of peak inspiratory flow by current preventer resistance at (A) RO and (B) R5. Horizontal bar of each box indicates median, whereas upper and lower edges of each box represent the upper quartile and lower quartile, respectively. The T-bar extends to 1.5 times the height of the box. An asterisk represents extreme outliers, which are values that are more than 3 times the height of the box. Pass rates for R5 were defined as 30 to $90 \mathrm{~L} / \mathrm{min}$ and for RO as 20 to $60 \mathrm{~L} / \mathrm{min}$, shown as dashed lines on each figure. Med, Medium.

pMDI (47\%). ${ }^{17}$ Insufficient inspiratory effort with the DPIs tested was associated with uncontrolled asthma in a multivariate model with other inhaler errors. Although "inspiratory effort not slow and deep" was not associated with uncontrolled asthma for pMDI users, other specific pMDI inhaler errors were. In our study, even patients familiar with the pMDI device failed the R0 test, illustrating the difficulties patients have with information retention with regard to inhaler device technique. Our data reinforce the importance of inhaler technique training with regular and repeated checks, including clear messaging with respect to the inhalation maneuver (DPI: Inhale quick and deep; pMDI: Inhale slow and steady). A recent review of inhaler technique critical errors in the context of asthma management reported that approximately $50 \%$ of patients fail to maintain correct inhaler technique over time and drew attention to the importance of patient psychosocial factors, such as motivation, for learning and maintaining good inhaler skills. ${ }^{18}$

Only $6 \%$ of all patients tested failed the R5 measurement. In this small number, the most significant independent risk factors for failing the test were female sex and age 70 years or more. Increasing age and female sex are 2 factors that have consistently been shown to be correlated with decreased PIFRs in patients with obstructive airway disease. ${ }^{7,19,20}$ The reasons for these findings have not been fully established, but others suggest they may include physiological factors such as reduced inspiratory muscle force. ${ }^{19,20}$ In this study, asthma severity was not significantly associated with the ability to pass at R5, also consistent with studies that have shown no correlation between PIFR and 
$\mathrm{FEV}_{1}{ }^{7,19,20}$ Patient characteristics significantly associated with the risk of failing the R0 measurement were male sex and having mild versus severe asthma, and a trend was also observed in younger patients failing versus older patients but this was not consistently shown across age groups. Although the reasons for this are not fully clear, one explanation may be that milder severity and younger patients with asthma more easily "overbreathe" (breath in too fast) compared with older patients and those with more severe disease. The patient's current preventer device had a significant influence on pass rates for both the R5 and R0 test, indicating that those used to using a pMDI were more likely to fail at R5 and those familiar with a DPI device were more likely to fail at R0, the latter being consistently shown across resistances R2 to R5, with the odds of failure increasing with increased device resistance. These findings suggest that a patient's inhalation maneuver is learned or imprinted on the basis of the device they are familiar with using. Certainly, others have suggested that past experience with inhalers is important in terms of maintaining correct inhaler technique and disease control. ${ }^{18,21-23}$ This further endorses the message that regular checks of inhaler technique and effective communication between health care providers and patients are an important part of asthma management. Training patients to perform correct inhalation maneuvers leading to activation of their device and optimal drug delivery requires health care providers to know the techniques required for each device. ${ }^{24}$ When selecting a suitable inhaler device for patients, it is also important that health care providers understand the concept that inhalation flow together with the resistance of the DPI provides the energy to cause drug deagglomeration and delivery of the whole dose to the lungs. ${ }^{24}$ Training aids such as the In-Check DIAL can aid suitable device selection.

A strength of this study is that we evaluated a real-world asthma population, with few exclusion criteria constraints, and therefore these findings would be expected to be largely generalizable. In this study, we did not evaluate how the inhaler resistance itself affected PIFR (by comparing PIFRs across different device resistances) but we have identified this as an area of future interest.

\section{CONCLUSIONS}

This study demonstrates in this adult asthma population that most patients can achieve adequate inspiratory flow to activate high-resistance DPIs (R5), suggesting that very few patients would be unable to use these devices. Nearly a third of patients fail to generate an inspiratory flow for correct pMDI use due to breathing in too fast, including a fifth of patients currently using a pMDI.

\section{REFERENCES}

1. Global Initiative for Asthma. Global strategy for asthma management and prevention. 2019. Available from: https://www.ginaasthma.org. Accessed March 9, 2020.
2. Biddiscombe MF, Usmani OS. Is there room for further innovation in inhaled therapy for airways disease? Breathe 2018;14:216-24.

3. Usmani OS. Choosing the right inhaler for your asthma or COPD patient. Ther Clin Risk Manag 2019;15:461-72.

4. Lavorini F, Usmani OS. Correct inhalation technique is critical in achieving good asthma control. Prim Care Respir J 2013;22:385-6.

5. Levy ML, Carroll W, Izquierdo Alonso JL, Keller C, Lavorini F, Lehtimäki L. Understanding dry powder inhalers: key technical and patient preference attributes. Adv Ther 2019;36:2547-57.

6. Azouz W, Chrystyn H. Clarifying the dilemmas about inhalation techniques for dry powder inhalers: integrating science with clinical practice. Prim Care Respir J 2012;21:208-13.

7. Ghosh S, Ohar JA, Drummond MB. Peak inspiratory flow rate in chronic obstructive pulmonary disease: implications for dry powder inhalers. J Aerosol Med Pulm Drug Deliv 2017;30:381-7.

8. Haidl P, Heindl S, Siemon K, Bernacka M, Cloes RM. Inhalation device requirements for patients' inhalation maneuvers. Respir Med 2016;118: 65-75.

9. Sanders MJ. Guiding inspiratory flow: development of the In-Check DIAL G16, a tool for improving inhaler technique. Pulm Med 2017;2017:1495867.

10. Lavorini F, Pistolesi M, Usmani OS. Recent advances in capsule-based dry powder inhaler technology. Multidiscip Respir Med 2017;12:11.

11. UK National Health Service. NHS Digital Read Codes. Updated April 1, 2016. Available from: https://digital.nhs.uk/services/terminology-and-classifications/ read-codes. Accessed May 11, 2020

12. Haughney J, Price D, Barnes NC, Virchow JC, Roche N, Chrystyn H. Choosing inhaler devices for people with asthma: current knowledge and outstanding research needs. Respir Med 2010;104:1237-45.

13. Lavorini F, Mannini C, Chellini E. Challenges of inhaler use in the treatment of asthma and chronic obstructive pulmonary disease. EMJ Respir 2015;3:98-105.

14. Papi A, Haughney J, Virchow JC, Roche N, Palkonen S, Price D. Inhaler devices for asthma: a call for action in a neglected field. Eur Respir J 2011;37:982-5.

15. Levy ML, Hardwell A, McKnight E, Holmes J. Asthma patients' inability to use a pressurised metered-dose inhaler (pMDI) correctly correlates with poor asthma control as defined by the Global INitiative for Asthma (GINA) strategy: a retrospective analysis. Prim Care Respir J 2013;22:406-11.

16. Giraud V, Roche N. Misuse of corticosteroid metered-dose inhaler is associated with decreased asthma stability. Eur Respir J 2002;19:246-51.

17. Price DB, Román-Rodríguez M, McQueen RB, Bosnic-Anticevich S, Carter V, Gruffydd-Jones K, et al. Inhaler errors in the CRITIKAL study: type, frequency, and association with asthma outcomes. J Allergy Clin Immunol Pract 2017;5: 1071-1081.e9.

18. Bosnic-Anticevich SZ, Cvetkovski B, Azzi EA. Identifying critical errors: addressing inhaler technique in the context of asthma management. Pulm Ther 2018;4:1-12.

19. Van der Palen J. Peak inspiratory flow through Diskus and Turbuhaler, measured by means of a peak inspiratory flow meter (In-Check DIAL). Respir Med 2003;97:285-9.

20. Malmberg LP, Rytilä P, Happonen P, Haahtela T. Inspiratory flows through dry powder inhaler in chronic obstructive pulmonary disease: age and gender rather than severity matters. Int J Chron Obstruct Pulmon Dis 2010;5:257-62.

21. Bjermer $\mathrm{L}$. The importance of continuity in inhaler device choice for asthma and chronic obstructive pulmonary disease. Respiration 2014;88:346-52.

22. Doyle S, Lloyd A, Williams A, Chrystyn H, Moffat M, Thomas M, et al. What happens to patients who have their asthma device switched without their consent? Prim Care Respir J 2010;19:131-9.

23. Thomas M, Price D, Chrystyn H, Lloyd A, Williams AE, von Ziegenweidt J. Inhaled corticosteroids for asthma: impact of practice level device switching on asthma control. BMC Pulm Med 2009;9:1.

24. Laube BL, Janssens HM, de Jongh FH, Devadason SG, Dhand R, Diot P, et al. European Respiratory Society; International Society for Aerosols in Medicine. What the pulmonary specialist should know about the new inhalation therapies. Eur Respir J 2011;37:1308-31. 